\title{
Estimating Morphological Features of Plant Growth Using Machine Vision
}

\author{
Himanshu Gupta, National institute of Technology Jalandhar, Jalandhar, India \\ Roop Pahuja, National Institute of Technology Jalandhar, Jalandhar, India
}

\begin{abstract}
Motivated by the fact that human visionary intelligence plays a vital role in guiding many of the agriculture practices, this article represents an effective use of machine vision technology for estimating plant morphological features to ascertain its growth and health conditions. An alternative to traditional, manual and time-consuming testing methods of plant growth parameters, a novel online plant vision system is proposed and developed on the platform of virtual instrumentation. Deployed in real time, the system acquires plant images using digital camera and communicates the raw image to host PC on Wi-Fi network. The dedicated application software with plant user interface, effective image processing and analysis algorithms, loads the plant images, extracts and estimates certain morphological features of the plant such as plant height, leaf area, detection of flower onset and fall foliage. The system was tested and validated under real-time conditions using different plants and leaves. Further, the performance of the system was statistically analysed to show promising results.
\end{abstract}

\section{KEYWORDS}

Computer Vision, Leaf Image Processing, Plant Image Processing and Analysis, Plant Morphology Assessment

\section{INTRODUCTION}

The human visionary and cognitive power to see, think, analyse and promptly take actions have motivated and accelerated research in the area of machine/computer vision systems over the last 50 years (Davies, 2012). Machine vision technology uses specialized devices such as image sensors/ cameras, image processing software tools and/or actuators to automatically capture, process and interpret a digital image of a real scene in order to extract useful information for monitoring, decision making, classifying and/or control of machines such as manufacturing robots or processes (Rosenfeld, 1985, Lewis, 2014). Machine vision is slightly different from computer-vision that concentrates on processing and analysis of digital image. Machine vision is a combination of image acquisition hardware, processing software and optionally the use of actuators for control of process/devices to perform image-based application specific tasks accurately, repeatedly and timely with visualization of information (Szelisk, 2010). With the advancements in affordable, sophisticated image sensors, smart camera and optics with digital interfacing, image processing methods and tools, the machine vision technology is fast evolving to solve complex application tasks (Teledyne, 2014). The highend aim of machine vision technology is to design systems and applications that can compete with the human visionary capabilities and perform tasks equivalent or better than humans in many cases (Teledyne, 2014). For e.g. computer vision system is effectively used in high speed production lines at a factory floor for detection of faults in manufactured components and sorting of materials. The work with vision system is done at much faster pace and accurately than the labour involved in the 
process (Lewis, 2014). In the case of medical imaging, machine-vision systems are a tool in the hand of doctors to obtain images of inner organs of the body, extract features and analyses images to provide better diagnosis, prediction and treatment of diseases (Chen, 2013).

Tools of machine vision have been extensively researched and used in broad range of applications for different tasks such as identification or recognition of objects, sorting and inspection of materials, health diagnosis, geographical and environment assessment etc. (Solari, Chessa \& Sabatini, 2012). Primary focus has been in the area of industrial automation and manufacturing for material inspection, electronic components inspection and guiding of robots for control operations using 2D or more recent 3D system (Tsarouchi et al., 2016), in medical image analysis and diagnosis ( $\mathrm{Li}, \mathrm{Ma}$, Wang \& Zhang, 2014), (Chen, 2013). Image processing with pattern matching techniques have been used in various applications such as character/handwriting recognition (Katungunya, Ding \& Mashenene, 2016), signature verification, sorting of materials based on different image attributes in food or production industry for quality assurance and analysis of satellite-based sensor images (Picon et al., 2012), (Key Technologies, 2012). Slowly and gradually, machine vision technology is spreading into other areas such as vehicle guidance or improve visibility during night and foggy weather, traffic monitoring and control (Li et al., 2018), (Singh, Vishnu \& Mohan, 2016). Machine vision technology is used for inventory control and management such as barcode reading, counting of articles etc., currency identification, defence, security and surveillance (Kar, Shrikhande \& Babu, 2016), crime investigation (Inspect, 2010), biometric measurement for personal identification, detection of diseases (Kallen, 2016), habitat monitoring of animals and birds, monitoring of agriculture fields and pest identification (Vázquez-Arellano, Griepentrog, Reiser \& Paraforos, 2016).

Motivated by the fact that human visionary intelligence plays a vital role in guiding many of agriculture practices and effective use of machine-vision technology for monitoring of plant growth related morphological parameters has not exploited much in the literature, this work is taken up. The aim is to devise a novel machine vision system for automatic identification of certain morphological parameters of a plant for its growth analysis. Specifically, to overcome some of the limitations of existing works, this paper focuses on implementing plant image processing methods for calculating certain morphological parameters related to plant growth cycle such as height of plant, detection of onset of flowers and fall foliage condition. Also, an improved method to estimate leaf area index with better accuracy is proposed. Moreover, to enhance the utility of this work, a complete vision system with camera and dedicated software tool (virtual instrument) named PIPME (plant image processing and morphological features estimation) with intuitive plant-user interface is proposed and implemented as a flexible multifunctional Virtual Instrumentation (VI) system. The software tool, developed by the authors, has image acquisition and processing algorithms to extract and analyse different morphological features and parameters of a plant. The tool has an interactive plant-user interface that is programmed to visualize the plant growth parameters online and/or analyse the results offline with data archive. Additionally, the system is tested in real-time situation and its performance is evaluated and validated. It is used to monitor the morphological parameters of a certain plant for a few days. Easy to use and operate, the tool can invariably be incorporated into a modern agriculture system to automatically provide vital information of plant growth parameters over its growing season, which is otherwise a tedious process and is generally performed manually.

This paper discusses the design, development, testing and validation of virtual instrumentationbased machine vision system with PIPME software tool (virtual instrument) for estimating and monitoring plant growth parameters. Using in-field digital camera, the plant images are acquired on PC, further processed and analysed using specific image processing algorithms implemented in PIPME tool to extract and estimate certain morphological parameters (leaf area, plant height, detection of onset of flowers, fall-foliage condition) with high level of accuracy and display the results on user interface.

The paper is organized into following sections: Section II reviews the state-of- the-art of sensing and vision systems in agriculture and surveys in detail applications of image processing and analysis to agriculture. Section III provides a brief background of plant morphology. Section IV and V discuss 
over-all design and development of proposed vision system and software tool for plant image processing and estimation of morphological parameters. Section VI describes the experimental work, results with performance evaluation and validation. The last section concludes about the salient features of the system and provides recommendation for future scope.

\section{RELATED WORK}

The state of the art of sensing system for agriculture extends from efficient use of smart sensor and/or network systems (Pahuja, Verma \& Uddin, 2013), (Ojha, Misra, \& Raghuwanshi, 2015) for monitoring environment and soil related parameters to intensive use of non-contact and nondestructive radiation or image based systems for crop management and analysis (Pandurng \& lomte, 2015) Further, these systems are integrated to ICT technologies such as IOT, cloud computing or mobile computing system for remote connectivity of agriculture system to end users for information access (Jayaraman et al., 2016). With the advancements in image acquisition and processing method $s$ there has been tremendous growth in development of plant image-based analysis systems to solve complex problems in the field of agriculture (Saxena \& Armstrong, 2014). Various types of image capturing methods such as satellite based remote sensing for regional monitoring systems (Susan et al., 2017), aerial/helicopter system with video camera (Shafian et al., 2018) has been used to capture crop image and analyze vegetative index to estimate crop yield, chlorophyll in crop (Yu et al, 2016). In-field use of camera on a network helps to acquire crop images with better temporal and spatial resolution. Various image analysis techniques have been used for crop management, diagnosis of plant diseases, identification of pests (Singh and Misra, 2017), identification of nutrient deficiencies from leaves analysis, quality inspection, sorting and grading of fruit etc. (Mahendran et al. 2011). For classification of agricultural products using image processing, machine learning methods have been employed. Further, machine learning (ML) and artificial intelligence (AI) techniques have been explored recently by many for knowledge intensive agriculture (Basnet and Bang, 2018).

Over a few decades, vision-based sensing system with image processing and pattern recognition techniques have been applied in the field of agronomy for different tasks. In some of the early works, image processing is used to detect faults in apple and peach using near infrared images (Crowe \& Delwiche, 1996). Sorting of apples based upon color grading using artificial intelligence technique is reported by authors in (Nakano, 1997), (Patel, Kar, Jha \& Khan, 2012). Use of machine vision tools for quality inspection of food products is reported by many. Authors in (Zhao \& Mi, 2010) proposed a relatively newer computer-based vision system for automatically grading of green hawthorn and forming quantitative standard for the same. Clustering analysis theory to sort out and classify area size of green hawthorn leaves in an industrial environment is discussed in this paper.

Focusing primarily on the applications of machine vision to plant growth assessment, this section, consolidates the key contributions for measurement of plant parameters using image processing techniques. Many authors have discussed the measurement of plant leaf attributes with different approaches. Authors in (Lü, Ren, Zhang \& Shen, 2010) discussed the method of new contour extraction to eliminate the influence of holes in the leaf image and applied pixel number statistics for leave area and leaf perimeter measurement. Authors in (Shivling, Singla, Ghanshyam, Kapur, \& Gupta, 2011) discussed image processing methods for calculating leave geometrical parameters such as length, width area and implemented MATLAB tool for the same. Sample of fifty leaves of various shapes and sizes were used in measurements and results were compared with existing graphical method like grid counting. Approaches to measure parameters and dimensions of specific cucumber and Bettal leaves were described by authors in (Tian \& Wang, 2009), (Nayak, Dey \& Sharma, 2015).

The concept of identifying plants species based upon specific traits of plant leaf was also exploited by many. Authors in (Xiao-dong \& Xiao-jie, 2009) proposed a new idea on feature extraction of a leaf based on visual consistency i.e. how to determine the direction in which to describe an object. The method was used to extract six shape features of leaf by rotating the leaf to a certain orientation 
with an improved inertia axis method according to human habit of observing an object. Another method to construct leaf features vector and classify plants using texture features of a plant leaf was described by authors in (Prasad, Kumar \& Tripathi, 2011). Another relatively newer technique based upon half leave image that provided an efficient way to recognize species was reported by authors in (Ulutuak \& Ugur, 2012). Area, extent and eccentricity features were extracted for each part and their proportions to each other were taken as new features to provide input to a probabilistic neural network (PNN) to classify the leaf samples. In one of the review works (Nathalie \& Breda, 2013), the authors discussed that it was most difficult to quantify leaf area index properly, owing too large spatial and temporal variability. Based upon the comparison of direct and indirect methods, indirect methods underestimate LAI, especially in forest stands. The accuracy, sampling strategy and spatial validity of the LAI measurements need to be assessed for quality assurance of both the measurement and modelling of all the LAI-dependent eco-physiological and biophysical processes of canopies. Another area of image-based leaf texture analysis to identify unhealthy plants was also researched. Authors in (Gavhale, Gawande \& Kamal, 2014), discussed a framework for leaf features extraction using statistical GLCM, and color feature extraction using mean values and a classifier method to inspect unhealthy area on leaf and identify disease.

Recently, the use of image processing methods for leaf area estimation using different objects as reference have been exploited by many. Authors in (You-wen et al., 2009) used a circle of known area as reference and took the image of leaf with that circle whereas the authors in (Lu et al., 2010) used a square of known side as reference and took the picture of leaf by placing it inside the square for calculating leaf area. In the paper (Nayak et al., 2015), authors calculated the number of pixels in one inch and used it to calculate the length and width of the leaf. Authors in (Soni, Dey \& Sharma, 2015) used a square of $1 \mathrm{~cm} \times 1 \mathrm{~cm}$ as reference and took the picture of leaf with that square to find leaf area.

Many of the researchers used typical features of a flower (reproductive structure found in plant) such as color, edge-detection, petals etc. to identify and classify plants species using image processing techniques. Author in (Tiay, Benyaphaichit \& Riyamongkol, 2014) used the edge and colour characteristics of flower images to classify the flowers and deployed Hu's-seven moment algorithm to acquire edge characteristics. The k-nearest neighbour was used to classify the flowers. The interactive flower image recognition system was proposed by authors in (Hsu, Lee \& Chen, 2010) and boundary tracking method was developed to extract flower regions as accurately as possible. Authors in (Das, Manmatha \& Riseman, 1999), provided the solution to the problem of indexing images of flowers. A new approach was proposed by them for indexing a specialized database of flowers by utilizing the colour and spatial domain knowledge available for the database. In (Najjar, Zagrouba \& Najjar, 2001) authors used a novel method to isolate the flower region from the background using OTSU's thresholding on lab colour space for segmenting flower region. In (Boykov \& Jolly, 2001), authors used graph-cut method which provided the technique to identify certain pixels as background or objects as segment part using contrast dependent prior Markov random field (MRF) cost function. Authors in (Fukuda, Takiguchi \& Ariki, 2008], proposed a method for identification of ROI (regions of interest) using the edges in the images that depend on the natural uncertainty principle. Author used C-Means algorithm to avoid ambiguity to decide the flower type and categorize the flowers based upon petals. Authors in (Hong, Chen, Li, Chi \& Zhang, 2004) proposed segmentation based colour clustering method to extract flower regions from flower images along with use of colour histogram and shape-based features set to characterize the flowers.

\section{PLANT DEVELOPMENT AND MORPHOLOGY}

The term plant growth analysis refers to a useful set of quantitative methods that describes and analyses the performance of plants grown in a natural, semi-natural or controlled conditions based upon measurement of certain morphological (external structure and features) parameters related to plant growth. Plant growth analysis is an explanatory, holistic and integrated approach to the 
interpretation of the form and function of plants and standard approach to the study of plant growth and productivity (Watada, Herner, Kader, Romani \& Staby, 1984). It uses simple raw data in the form of weights, areas, volumes and content of plant components, to examine the processes within the plant leading to plant growth. Plant growth analysis concept has proven to be highly effective and successfully applied to other related areas such as study of the reaction of certain kinds of plants to different environmental conditions, research on cultivation/management practices for better plant productivity, design of plant growth model or study events that can make the plant more or less productive (Kuchay \& Zargar, 2016).

Plant growth and development is the process by which a structure from seed originates and mature as a plant with new organs to produce yield. Growth of the plant is attributed to interaction of physiological processes such as respiration, photosynthesis and transpiration dependent upon environmental and soil variables that results in changes in its internal structure and external physical form during different stages of its growth. At the molecular level, plant growth involves an increase in cell number by cell division, cell enlargement, formation of hormones and tissues. Externally plant growth process results in physical changes in its structure, form and features with formation of new organs (Hopkins, 1999). Typically, plant growth and development pattern/process are divided into three parts: the growth, differentiation and development involving qualitative and quantitative changes in plant structure. The plant growth is an indeterminate phenomenon that includes permanent increase in the size of tissues and organs, as well as weight and indicates quantitative change in the plant overall structure. The differentiation is the actual formation of tissues and organs in plant to depict qualitative changes in plant structure. The development is the overall growth and differentiation of plant organism as the time goes by. Different stages of plant development is broadly summarized as: fertilization $\rightarrow$ gemmule $\rightarrow$ seed $\rightarrow$ germination $\rightarrow$ seeding $\rightarrow$ growth $\rightarrow$ flowering $\rightarrow$ fruit $\rightarrow$ aging (Hopkins, 1999)

Mathematically, the plant growth process is represented by sigmoid curve as shown in Figure 1(a) (Lin et al., 2003). The sigmoid curve representing the plant growth process is divided into three stages: an initial stage with slow growth, middle stage and end stage in which growth rate slows down. The initial stage is the period when the plants set their bodies after sprouting with stored nutrients. In the middle stage, the plants shoot system develops with crowding of new shoots (branches) and leaves, formation of organs such as buds, flowers and fruits. Biosynthesis and photosynthesis processes occur rapidly at the time. In the final stage, plant growth is slowed down. There is hardly increase in the height of the plant and will compete for metabolites, water, minerals and light, as well as the accumulation of growth inhibitors.

Study of plant development specifically based upon its external structure, features and forms is referred to as plant morphology or phytomorphology. Phytomorphology is useful in visual identification of plants and deals with quantitative and quality analysis of different physical parts or features of plants organs such as roots, shoot, stems, leaves, flowers, seeds, fruits etc. to provide growth assessment. Figure 1 (b) shows a typical plant structure with different morphological parts with associated features and their parameters/attributes (Schopfer, 2006). A plant is differentiated into an underground root system and an aerial shoot system with distinct morphological organs. The shoot system consists of both vegetative parts such as stem, branches, leaves and reproductive parts such as flowers and fruits.

All the visible structural components of the shoot system that provide information of growth, yield, health status etc. are morphological characters. Further, morphological characters are represented by set of features defined by measurable attributes/parameters. Morphological features that can be compared, measured or counted are known as quantitative characters whereas others that indicate quality of the plant such as leaf colour, leaf texture, fruit colour, pubescence etc. are referred as qualitative morphological characters. For example, size of flower petal is a quantitative feature of the plant organ flower. It is measured in terms of certain attributes such as width, length, area, perimeter etc., commonly referred to as descriptive parameters. Such features and parameters are used to assess the differences or similarities in plant taxa for plant identification, classification and growth analysis. 


\section{PROPOSED PLANT VISION SYSTEM}

Generally, visual inspection of plant reveals certain changes in the plant structure, form etc. that provides the basis to access plant growth and health and this method can effectively be automated using image processing and machine learning algorithm. The machine vision system that captures, processes and analyses the plant images to provide valuable information about plant is referred as plant vision system. This work proposes the design and development of a computer (PC) based plant vision system using Virtual Instrumentation technology for real-time monitoring and analysis of specific multi-morphological features and parameters of plant such as leaf area, plant shoot length, detection of flowers, fruits and fall foliage. These features are often required to analyse growth and health status of plant and are of valuable important in the field of agriculture and zoology. In the traditional agriculture set-ups, many of the plant morphological parameters features are manually inspected by humans on day to day basis and recorded for further analysis using conventional methods such as meter scale, grid counting graphical method for leaf area or using hand-held instrument such as leaf area meter (Li-COR, 1988). Though the methods are accurate, but the procedure is manual, tedious, time-consuming and does not support automatic online measurement of plant growth parameters. As an alternative to existing methods, a semi/fully automatic plant image acquisition and analysis system to estimate plant growth related certain morphological parameters of plant is implemented and discussed in this paper.

Figure 2 shows a typical architecture of proposed PC- based plant vision system to estimate certain morphological parameters of a plant. Based upon Virtual Instrumentation technology (National Instruments, 2000a), the system uses a suitable camera as vision sensor that is interfaced to general purpose computing platform such as PC. The computer is equipped with dedicated application software (virtual instrument) tool, named PIPME (plant image processing and morphological features estimation) that defines all the functions and features of the system. The plant images

are captured by the suitable camera (low resolution Mi4i mobile camera or high-resolution Nikon D3300 DSLR camera) (Nikon, 2014) fixed at the appropriate height on the tripod stand. The plant image frames as captured by the camera are forwarded wirelessly on the Wi-Fi network to the host PC. The acquired images are processed and analysed by PIPME to estimate growth related morphological parameters of the plant and display the results on graphical user interface.

Figure 1. Plant development (a) Sigmoid curve depicting plant growth process (b) A typical plant structure depicting morphological features (Lin et al., 2003).
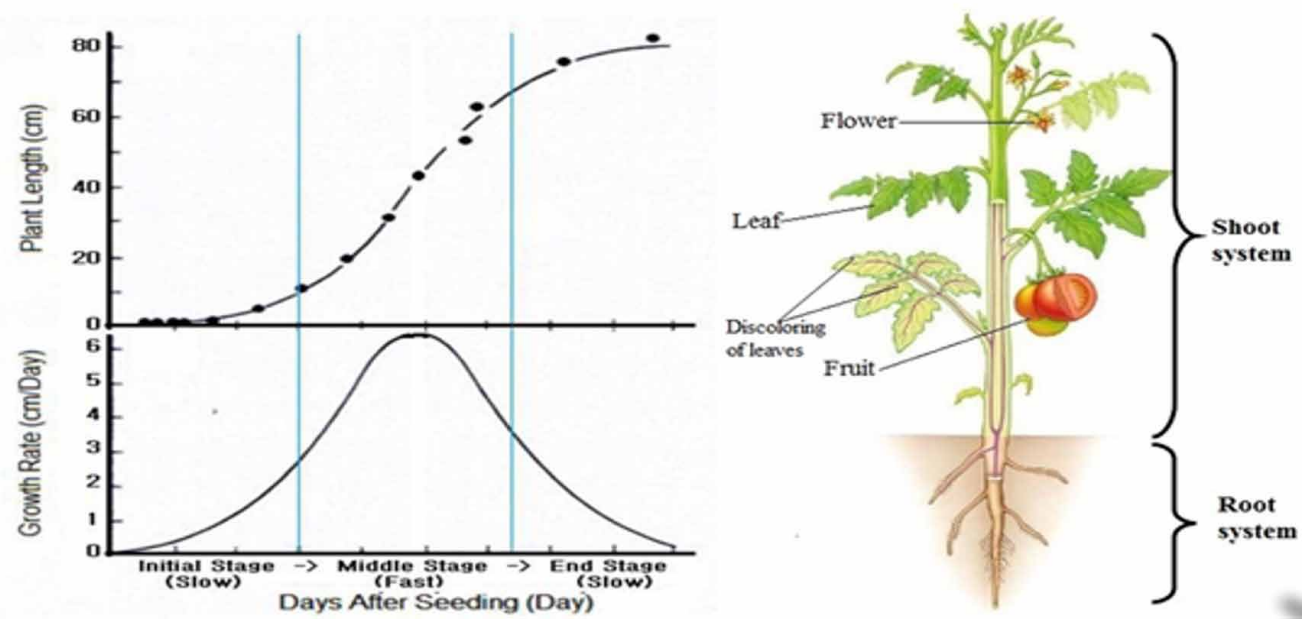


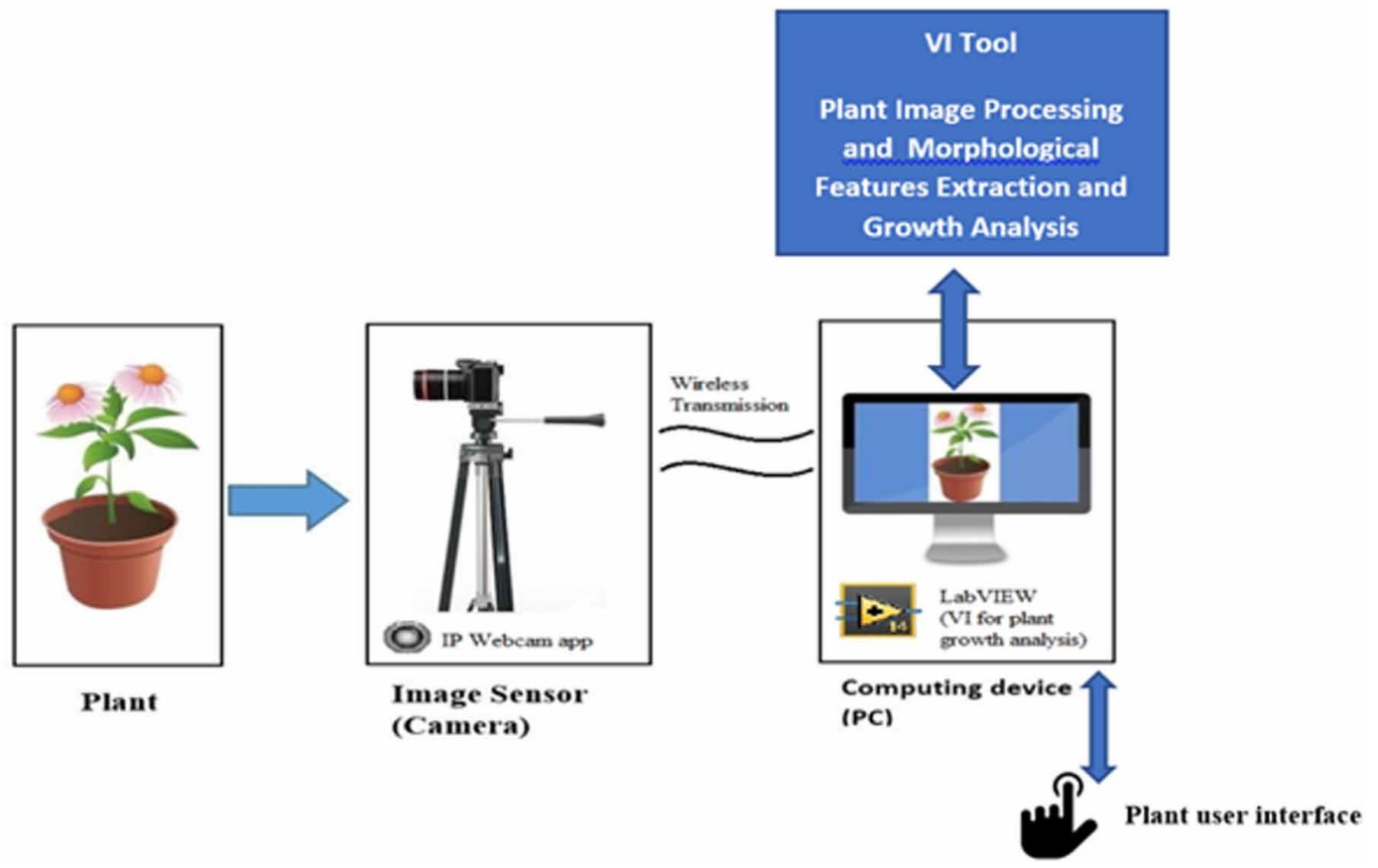

\section{TOOL FOR PLANT IMAGE PROCESSING AND MORPHOLOGICAL FEATURES ESTIMATION (PIPME)}

All the functions and features of the proposed plant vision system are defined by the dedicated software virtual instrument tool, named PIPME (plant image processing and morphological features estimation). It is an integrated application program that acquires, processes plant images for feature extraction and estimation of certain morphological growth parameters of plant in offline and online mode. Programmed on the standard platform of LabVIEW (National Instruments, 2000a, 2000b), (Johnson \& Jennings, 2011), a graphical programming language from National Instrument, the tool is developed with an interactive menu-based Plant- user interface (PUI) with unique functions and features. Figure 3 shows the functional design model of plant image processing

VI tool with well-designed user interface. The interface allows the user to operate the system, acquire and load plant images, estimate multiple growth parameters of the plant using suitable image processing and analysis methods and visualize the parameters of a plant graphically with absolute date-time stamp. The section below explains the important functional modules of the VI tool.

\subsection{Online Plant Image Acquisition}

This functional module with the user interface allows the user to capture plant images using a camera. For online image acquisition, the camera is wirelessly interfaced to PC on Wi-Fi network. The Wi-Fi enabled camera is pre-configured to work on the same IP network with the host PC. The PC acts as a server for mobile camera. Figure 4 shows the flow chart of image acquisition program that controls the operation of camera and streaming of digital image frames over Wi-Fi network to PC. The camera operates under automatic or semi-automatic modes. In automatic mode, the camera is automatically triggered after twenty-four hours to take the multiple plant image frames on each day whereas in the case of semi-automatic mode the user starts the plant image acquisition process. At the click of START button on the user interface, the program actively opens the camera, load the configuration 
Figure 3. Functional design model of VI Tool (PIPME) for plant image processing and estimation of morphological features

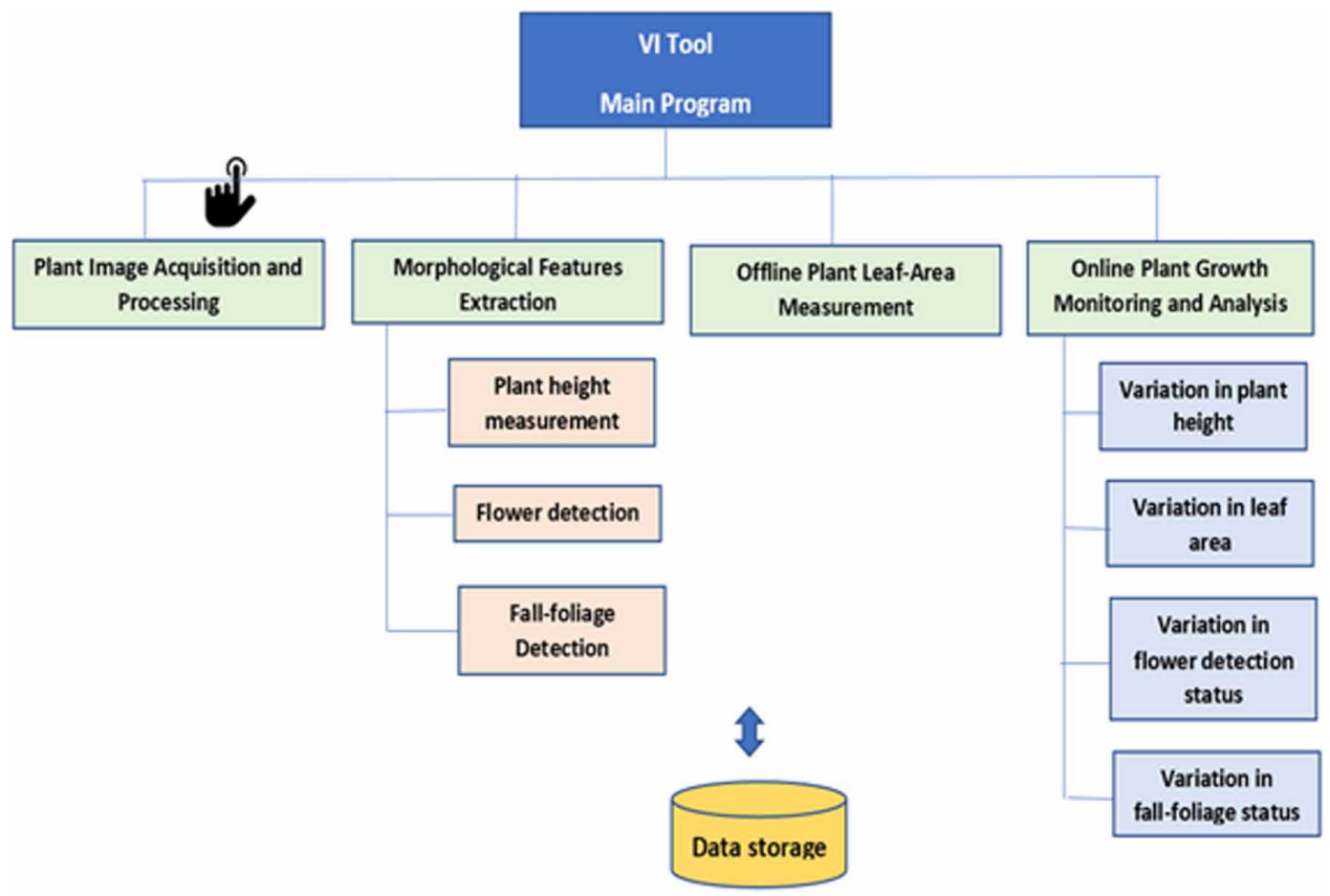

file, creates an image buffer in PC memory with unique name and snaps plant image data frame at low-speed to display on the panel. Multiple frames are captured at the rate of $2 \mathrm{~s}$ and simultaneously logged in the files, dynamically created with date and frame number stamped. The image pixel data is stored in .JPG format (Relf, 2004). The program is interactive with the prompt of the message box that provides an option to restart the process again if image capture is not proper.

\subsection{Morphological Features Estimation}

This functional module processes the digital image of the plant, extracts and estimates important morphological features representing vegetative and reproductive organs of plant such as height/length of shoot system, area of leaf, detection of onset of flowers and fall foliage condition and display the results on user interface. Figure 5 shows the flow chart of algorithms for estimation of plant morphological features for growth analysis. The algorithm operates on the selected pre-stored plant images for the particular day to estimate the average value of the different morphological parameters of the plant for the day. Further, each day measured parameters are logged in a file with day-stamp. The description of algorithms for plant image processing, extraction and estimation of growth-related morphological features of plant using non-destructive machine vision methods are explained below:

\subsection{Estimating Plant Height}

The height of plant (shoot length) is the distance between the base (reference level: soil/pot) and the highest point of the plant when all the parts of the plant is in its natural position. It is one of the important external quantitative morphological parameter that is measured to ascertain the growth rate or annual growth cycle of plant of same/ different species, grown in different /same weather or nutrient conditions. It is generally accounted for interpretation of characteristics of a plant such as growth, vigor etc., based upon the comparison of growing conditions such as weather, glazing, 
Figure 4. Flow chart of image acquisition program using Wi-Fi enabled digital camera

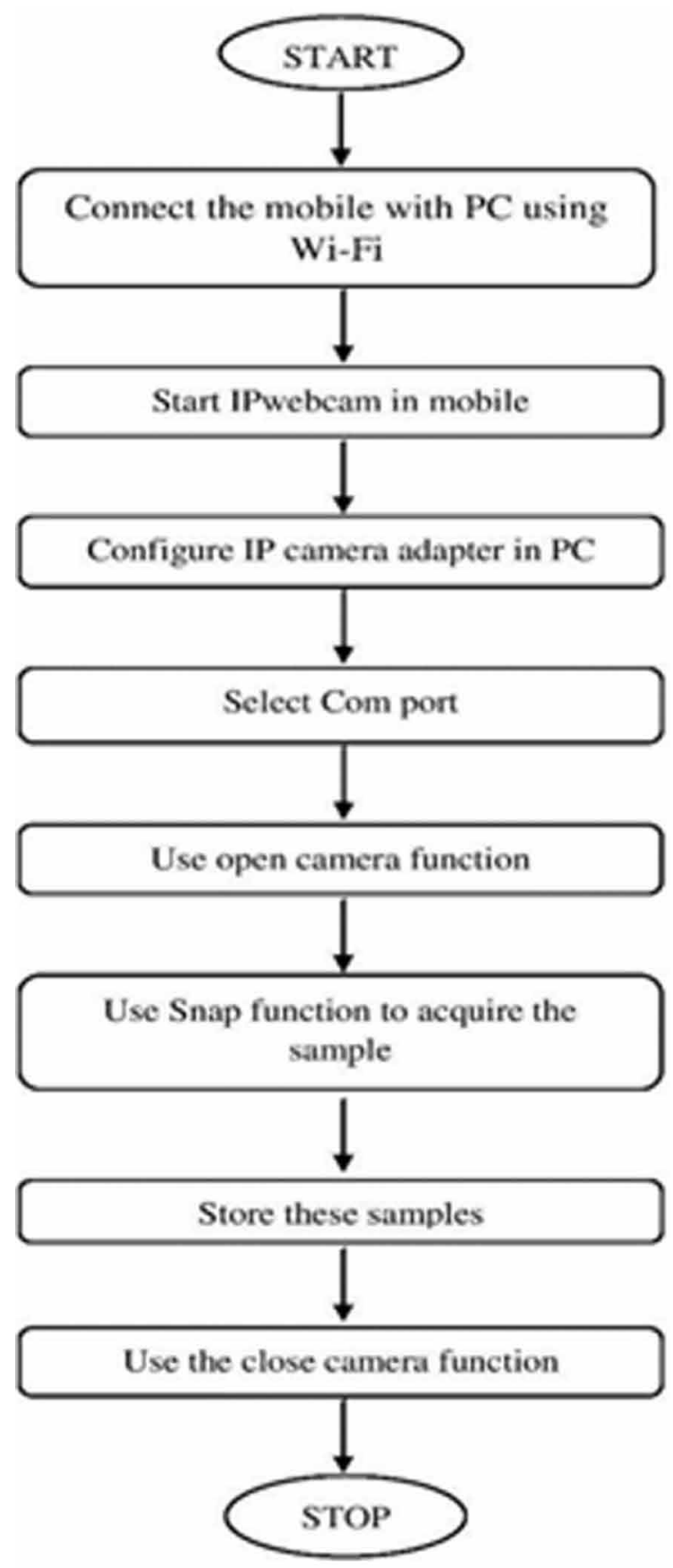

growing media (soil/soilless) with respect to crop inputs or treatment such as water and nutrient level, use of fertilizers, seed culture, etc. (Heady, 1957).

The proposed plant vision system measures the height of the potted plant based upon calculating and mapping the pixels representing the plant height in the acquired image using color discrimination 
and subtractive method. For estimating height of potted plant, the user selected plant image is loaded, displayed and duplicate copy of the same is also created (Figure 5). Both the images are processed in parallel to find the shoot length (Relf, 2004). Since the plant with the pot presents different color, one image is processed in gray-scale and other is processed as color image using threshold method. From the first image, color plane is extracted to convert the image to gray scale using RBG color model and then the image is converted into binary using two-level threshold method, a commonly used technique for image segmentation to highlight regions of interest (Crane, 1997). In the simple two level threshold technique, to separate one object from the background, the suitable threshold value is chosen from plant image histogram and then based upon the threshold level of intensity the plant image is modified (Russ \& Heal 1999).

Similarly, the second color image of the plant is converted into binary with pot color (red) as threshold intensity to obtain the binary image of pot in the processed image. This way, one of the processed image represents the pictorial information of full plant while the second processed image represents the pictorial information of the pot used. Further, from the binary images, border objects and small particles representing noise are removed using open binary morphological operation such as erosion followed by dilation to improve image quality (Crane, 1997). The two binary images are separately processed using logical operations on rows and column pixel values. All the column data is logically OR and then the row data is summed thus providing the count of vertical pixels representing the total plant height and pot height for the two images. The difference of pixels count in two cases is obtained to get the pixels corresponding to plant height. The shoot length of the plant is then calculated by multiplying the pixel count by reference value of height per pixel. The height per pixel value is experimentally calculated by using the pot image data and actual measured value of the pot.

\subsection{Flower Detection}

A flower, sometimes known as a bloom or blossom, is the reproductive structure found in plants that are floral. The biological function of a flower is to effect reproduction, usually by providing a mechanism for the union of sperm with eggs. After fertilization, the ovary of the flower develops into fruit containing seeds. For flowering plants, generally recording the number of days since initial planting to the first flower is an important parameter to indicate the growth and health conditions of a plant (Hopkins,1994). Detection of flowers is also considered an important parameter while analyzing the effect of some of the controlled environmental conditions on plant development. Recently, based on image processing, many flower recognition system using typical features such as color, edgedetection, petals etc. were proposed by many as discussed in section II.

The proposed plant vision system incorporates a method (Figure 5) to detect the presence or absence of flowers in the image of the plant using pattern/templet matching algorithm. The algorithm matches the pattern using 'linear spatial filtering' method that scans the image at all the possible positions with respect to the templet (Pakhira, 2011), (National Instruments, 1999a). The templet is called the filter mask. The center (or the origin) of the template $\mathrm{T}(\mathrm{xt}, \mathrm{yt})$ is moved at each $(\mathrm{x}, \mathrm{y})$ point in the search image $S(x, y)$ and the sum of products between the coefficients in $S(x, y)$ and $T(x t, y t)$ over the whole area spanned by the template at each reference pixel of the search image is calculated (Pakhira, 2011). The positions with the highest score or very near to the highest score are the matched templet positions. The matched parts are circled and counted to indicate the detection of flowering condition. If the count is zero, onset of flower is not detected. By analyzing the images of the plant for subsequent days, the detection date for onset of flower can be ascertained.

\subsection{Fall Foliage Detection}

Fall foliage refers to the condition of changing of leave color of the plant due to changes of weather in autumn reasons. This is one of the qualitative features that indicates the health status of the plant. In this work, detection of fall-foliage condition refers to change of healthy green color leaf to yellow (pale) due to moisture stress in plant because of over and under watering condition (Schopfer, 2006). 
Figure 5. Flow chart of image processing algorithms for estimation of plant morphological features

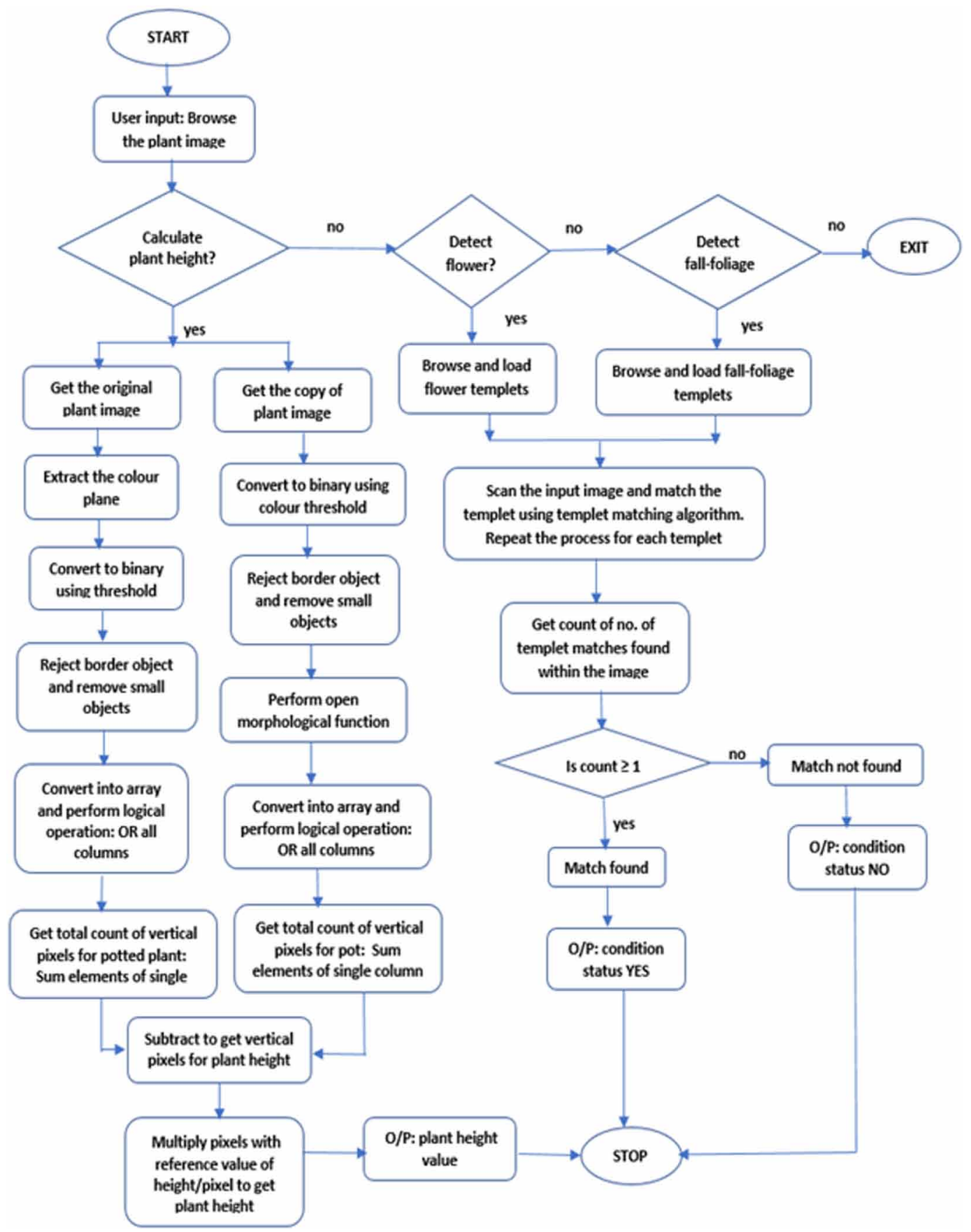

This may also be due to environmental changes or lack of proper nutrients to the plant. In autumn season, this phenomenon is natural in plants, but in other seasons this may be of concern to the grower. Early detection of such kind of condition will help the grower to safeguard plant's health by timely regulating plant growing conditions. 
The proposed vision system has the novel feature (Figure 5) of detecting fall foliage condition of leaves turning yellow in the plant image and generating an early warning signal for the same to take appropriate measures if the condition is abnormal. The plant image to be scanned is loaded and templets of fall-foliage leaves are automatically selected and compared one-by-one with the input plant image using templet matching algorithm based with linear spatial filtering using cross-correlation value (Crane, 1997), (National Instruments, 1999b). If the cross-correlation value, in any of the cases, exceeds the threshold value then the match is found, matched parts are circled and counted. If the count is non-zero, fall-foliage condition is detected, otherwise no fall-foliage has occurred, indicating thereby that the health status of the plant is OK. By analyzing the images of the plant for subsequent days, the real status of fall-foliage condition within the plant can be estimated.

\subsection{Leaf Area Measurement}

Leaf is an important organ and morphological feature of a plant. Plant leaves are real world entities, always have changes in physical appearance (Goudriaan \& Monteith, 1990). Due to large spatial and temporal variability in leaf size and shape of plant species, it becomes difficult to quantify the leaf. It is generally characterized by measurement of many parameters/attributes such as leaf dimensions, size, area, eccentricity, color, texture etc. These set of parameters provide quantitative assessment of plant leaf and that forms the basis to identify plant, judge the quality of leafy vegetables or provide indication of plant growth. (Kuchay et al., 2016). Area of the leaves is one of the key feature of the plant that not only characterizes the plant species, but is used as an index to analyze growth and development. Measuring the leaf area of plants spans many scientific disciplines. Observing the changes in leaf area is important for assessing growth of different plants on the planet. It is basically important as a parameter in development of different growth and climate models. This variable represents the amount of leaf material in ecosystems and controls the links between biosphere and atmosphere through various processes such as photosynthesis, respiration, transpiration and rain interception (Demin, Wei \& Lijing, 2012). It is an important parameter in ecophysiology, especially for scaling up the gas exchange from leaf to canopy level (Jingwen \& Hong, 2012). Leaf area measurement also provides information about Leaf Area Index (LAI), defined as amount of one-sided leaf area per unit area of ground and is a term generally used in many specific fields of plant growth modeling etc. (Lin et al., 2016). LAI is used to predict photosynthetic primary production, evapotranspiration and as a reference tool for crop growth (Goudriaan \& Monteith, 1990). Leaf area is generally inferred by many of the existing methods such as direct destructive methods including grid counting method and paper weighing method (Nathalie \& Breda, 2003). Using indirect means, electronic leaf area meter e.g. LI-3000A of Licor company (Li-COR, 1988) provides reading of leaf area on the display unit. Also, use of image processing methods for leaf area estimation have been exploited by many (section II).

The proposed technique for leaf area measurement combines the direct method of grid counting with image processing to provide better estimate of average leaf area of a plant as compared to the existing image processing methods. The method uses white grid paper background to take leaf image. A grid paper with vertical and horizontal lines having 41 columns and 85 rows with square block of $2 \mathrm{~mm} \times 2 \mathrm{~mm}$ area and total squares of 3485 is accurately and precisely prepared. The leaf is placed on the grid paper and the image for the same is captured under full brightness condition by high definition camera such as DSLR camera having 24MP (mega pixel) resolution. Likewise, the individual leaf images of number of leaves of a plant are captured and interfaced to PC. Each leaf image is stored in the file with date, leaf and plant label for further processing and analysis.

Figure 6 shows the flow chart of the algorithm for leaf area measurement. The captured image of leaf with grid background follows sequence of digital image processing and analysis steps to estimate the leaf area. The stored leaf image of the plant is read and firstly the brightness and contrast of the color picture of plant leaf is improved upon using the appropriate adjustment to pixel intensity based upon brightness and contrast values that vary in the range -255 to 255 . The modified color image is converted it to 8-bit monochrome/grayscale image using the additive color mixing model based 
upon luminance perception of the human eye (Crane,1997). The gray image is inverted and converted into binary using threshold to highlight the regions of interest in two different color. Thereafter, to improve image quality, advance morphological operations are performed on the image. Using simple morphological operations such as erosion

$\mathrm{B}_{\mathrm{L}}=\mathrm{B}_{\mathrm{T}}-\mathrm{B}_{\mathrm{v}}$

$A_{L}\left(m m^{2}\right)=B_{L} \times 4$

\subsection{Online Plant Growth Analysis}

This functional module monitors the variation in the growth parameters estimated by image processing methods for a particular plant over subsequent days. Corresponding to each day, plant image is acquired and stored by the system online, growth parameters are calculated as described in subsections above and logged in a file. For the given plant index, this module loads the parameter file that contains the estimated values of growth parameters such as leaf area, plant height, status of flower and fallfoliage detection for subsequent days. Further, the data file is processed to display day variation in each plant growth parameter on respective waveform graphs. This automatic graphical representation of growth parameters of a plant on continuous absolute time-scale helps the experts to judge plant growth and health conditions based upon the comparison with some standard recommendations given by researchers/consultants in this area. This helps the grower to take timely action to improve the growth rate and protect plant from any disease or weak health conditions if adequate overall plant growth is not indicated.

\section{RESULTS}

To analyze the working performance of the proposed plant vision system and validate the results of image processing algorithms for estimating different growth parameters of the plant, many experiments were conducted. Online tests were done on the same and/or different plants, randomly on different days and/or continuously for some days. The plant images were captured by DSLR or smart mobile phone camera with high resolution. A few of the preliminary experimental work and results are discussed in subsequent sections.

\subsection{Online/Offline Plant Image Acquisition and Processing}

The proposed plant vision system was installed with the camera (Nikon D3300) interfaced to PC running the plant-vision software (VI) tool. The camera was placed on the tripod stand to take the full image of the 'test' plant in the pot (Figure 2). Many plant images were taken on each day and / or subsequent days to online process and analyze the growth parameters. The online acquired plant image samples were used to extract certain growth parameters such as plant height, flower detection and foliage detection in a non-contact manner without disturbing the natural growth process of the plant. For leaf area measurement, certain leaves of the 'test' plant were plucked and images of the same were offline acquired and stored in PC for analysis by the software.

\subsection{Online Plant Image Acquisition}

For online plant image acquisition, the camera of the vision system captured the digital image of the 'test' plant and interfaced it to PC for the VI tool to acquire and process it further. Figure 7 shows the front panel of 'online image acquisition' VI. At the click of 'Start acquisition' button, the program acquired the images of the plant continuously at the interval of 20 seconds. Each image was displayed 
Figure 6. Flow chart of image processing algorithm for measurement of leaf area of a plant and dilation, the image is further improved upon by removing the small particles, border objects, fill the holes, to get the final processed image. The image is then inverted to get the final processed image (Relf, 2004), (National Instruments, 1999). The processed image is analyzed to estimate the leaf area. For this, the number of the visible grid blocks $\left(B_{v}\right)$ are counted using square detection operation. Then the number of square blocks covered by the leaf $\left(B_{L}\right)$ are calculated using equation (1) as the number of total blocks $\left(B_{T}=3485\right)$ on the grid are known. The area of the leaf $\left(A_{L}\right)$ is hence calculated using equation (2), the area of each block being $4 \mathrm{~mm}^{2}$.

\section{Capture the image}

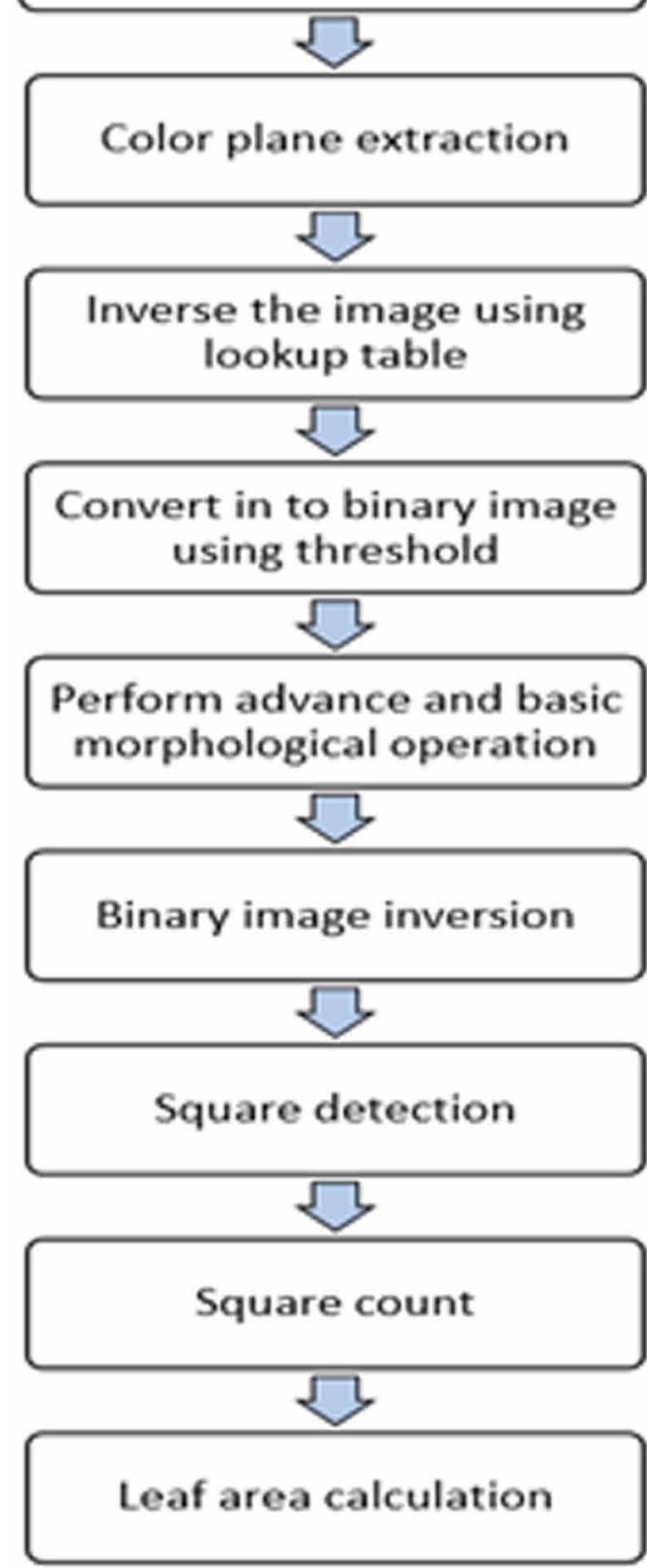


on the indicator. When the fixed number of images (pre-defined) of the plant were acquired, a message box interactively allowed the user to STOP acquisition. The acquired images were dynamically stored in the file with date/time stamp.

\subsection{Online Growth Parameters Extraction and Estimation}

The acquired plant images were processed and analyzed using 'growth parameter extraction' menu button on plant user interface of the VI tool. Figure 8 shows the front panel of 'growth parameter (morphological features) extraction' virtual instrument that indicates the average estimated values of plant height, number of fall foliage (color matches) and number of flowers detected for the captured plant image for the specific day. Based upon the selected input date, multiple samples of plant images for the day were reloaded and sequentially processed by the specific image processing algorithms to display average value of plant morphological parameters on respective indicators. The estimated results were in accordance with observed results. As obvious from plant image, there was no trace of flowers and also one of the leaf of the plant was getting pale (changes in color from normal green) hence fall foliage condition was detected. The average plant height value of $10.01 \mathrm{~cm}$ as displayed on the panel was measured with high accuracy. The reading was

\subsection{Offline Leaf Image Acquisition and Leaf Area Calculation}

Images of multiple leaf samples of a 'test' plant was taken by placing each on a white grid background using high resolution camera. The images were transferred to PC and saved in an appropriate file with date index for further processing by the VI tool. Figure 9(a) shows the front panel of offline leaf area measurement VI. Based upon the user input date, the corresponding leaf images for the day were processed sequentially by the underlying image processing algorithm for leaf area calculation. The measured average value of leaf area of $1000 \mathrm{~mm}^{2}$ was displayed on the indicator. Raw and processed sample leaf images were also indicated. The reading on the panel was validated by comparing the same with the manually measured average leaf area value of $1002 \mathrm{~mm}^{2}$ using graphical grid counting method. having very low value of $2 \mathrm{~mm}$ error in the reading. Many such tests were conducted and overall mean percentage error in estimating the leaf area remained very low $(0.48 \%)$ in comparison to other cited works as shown in Figure 9(b).

\subsection{Growth Analysis and Data Validation of a Single Plant}

The main goal to develop the proposed plant vision system is to deploy the system for continuous, automatic online acquisition, monitoring and analysis of growth-related morphological features of 'test' plant in the agriculture field on daily basis. Along with monitoring of the parameters using plant vision system, each parameter was measured with standard measuring instrument to ascertain the actual value of parameters and henceforth validate the proposed algorithms and working performance of the system. The vision system was installed near the 'test' plant which was naturally growing in open area and was watered properly. Each day multiple images of the plant were acquired, logged and processed to display the average value of growth parameters on the respective user interfaces. Also, for each day, actual values of plant growth parameters were recorded to validate the estimated values and analyse the percentage error in the measurement process. Table 1 summarizes the estimated and actual values of morphological features of a 'test' plant along with statistical analysis of data. Figure 10 (a), (b) and (c) show variations in estimated and actual values of plant height, leaf area over the 15 days of continuous monitoring along with the respective error curves. During these days, the plant height and leaf area increased gradually. Plant height and leaf area varied from $24.6 \mathrm{~cm}$ to $33.6 \mathrm{~cm}$ and $980 \mathrm{~mm}^{2}$ to $1095 \mathrm{~mm}^{2}$, respectively. Both the parameters were monitored with high level of accuracy. The error in plant height remained low, varied in the range -0.4 to $+0.3 \mathrm{~cm}$ with mean percentage error of $-0.02 \%$ and root mean square percentage error of $0.69 \%$ respectively. For leaf area, the error also remained low, varied in the range 2 to $9 \mathrm{~mm}^{2}$ with mean percentage error of $0.48 \%$ and root mean square percentage error of $0.2 \%$. 
Figure 7. Front panel of 'online image acquisition' VI to capture, display and store the plant image acquired by digital camera interfaced to PC validated by comparing the same with the manually measured shoot length value of $10.58 \mathrm{~cm}$ using standard measuring tape.

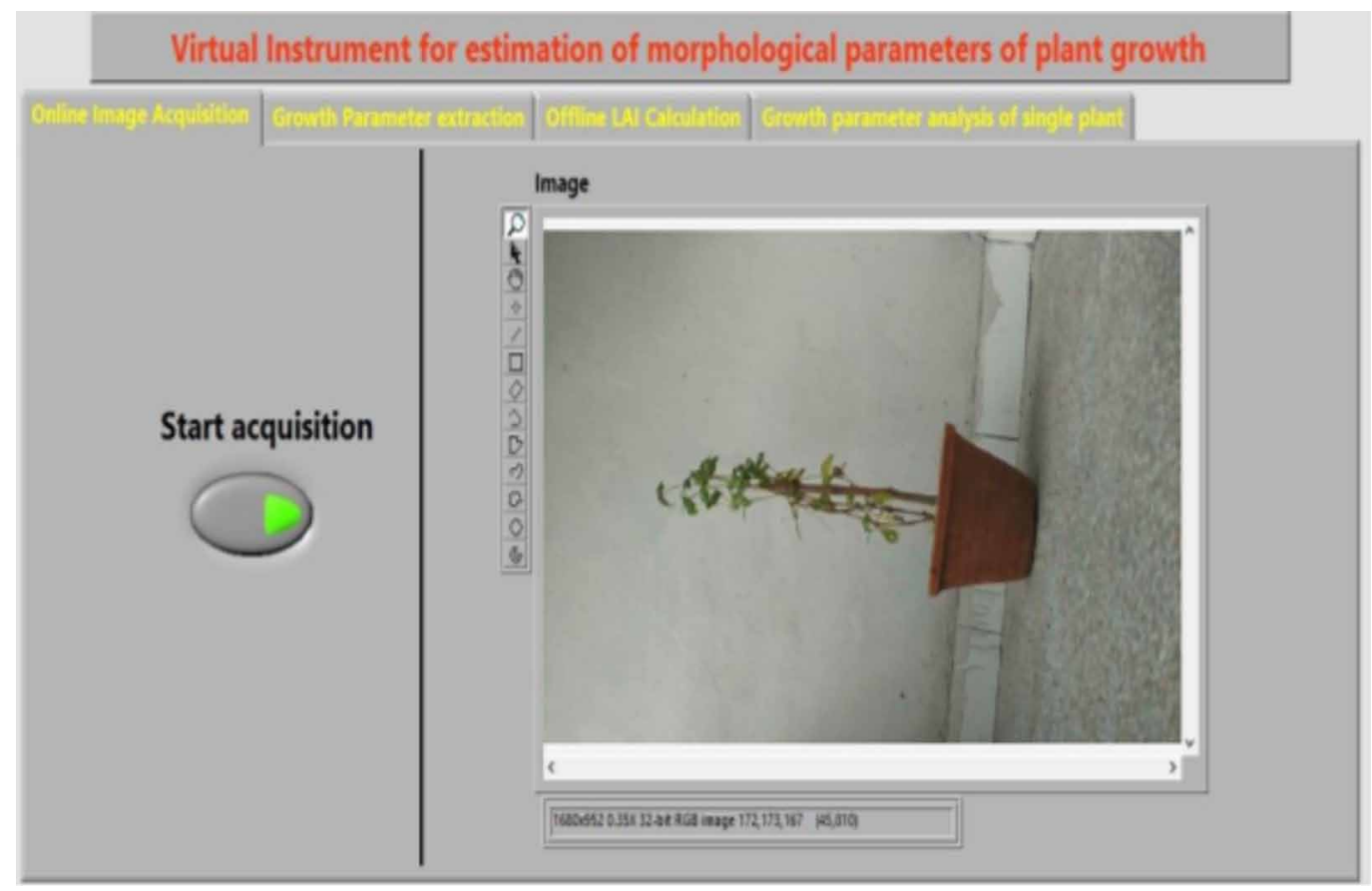

Figure 8. Front panel of 'growth parameter (morphological features) extraction' VI that indicates the average estimated values of plant height, number of fall foliage (yellow color matches) and number of flowers detected for the plant image of specific day

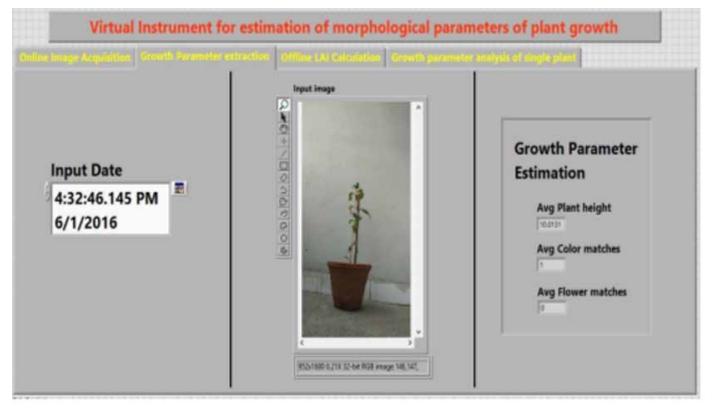

Figure 11 (a) and (b) indicate the statistical test results and performance metrics of fall foliage and flower detection algorithms based upon the actual and estimated/indicated status of each during the days. The probability of detecting the number of true cases as positive (true positive: TP) or number of false cases as negative (true negative: $\mathrm{TN}$ ) remained very high as compared to false indication (false negative: FN or false positive: FP). Further, the performance metrics based upon the combinational test statistics of the detection algorithms such as sensitivity specificity, predictive value true and predictive value false were evaluated using equations (3), (4), (5) and (6) (Parikh at al., 2008), respectively. 
Figure 9a. Front panel of plant leaf area measurement $\mathrm{VI}$

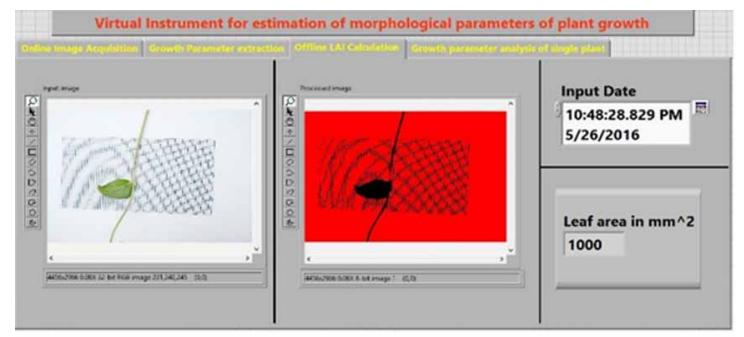

Figure 9b. Comparison of percentage error in leaf area measurement with other cited works

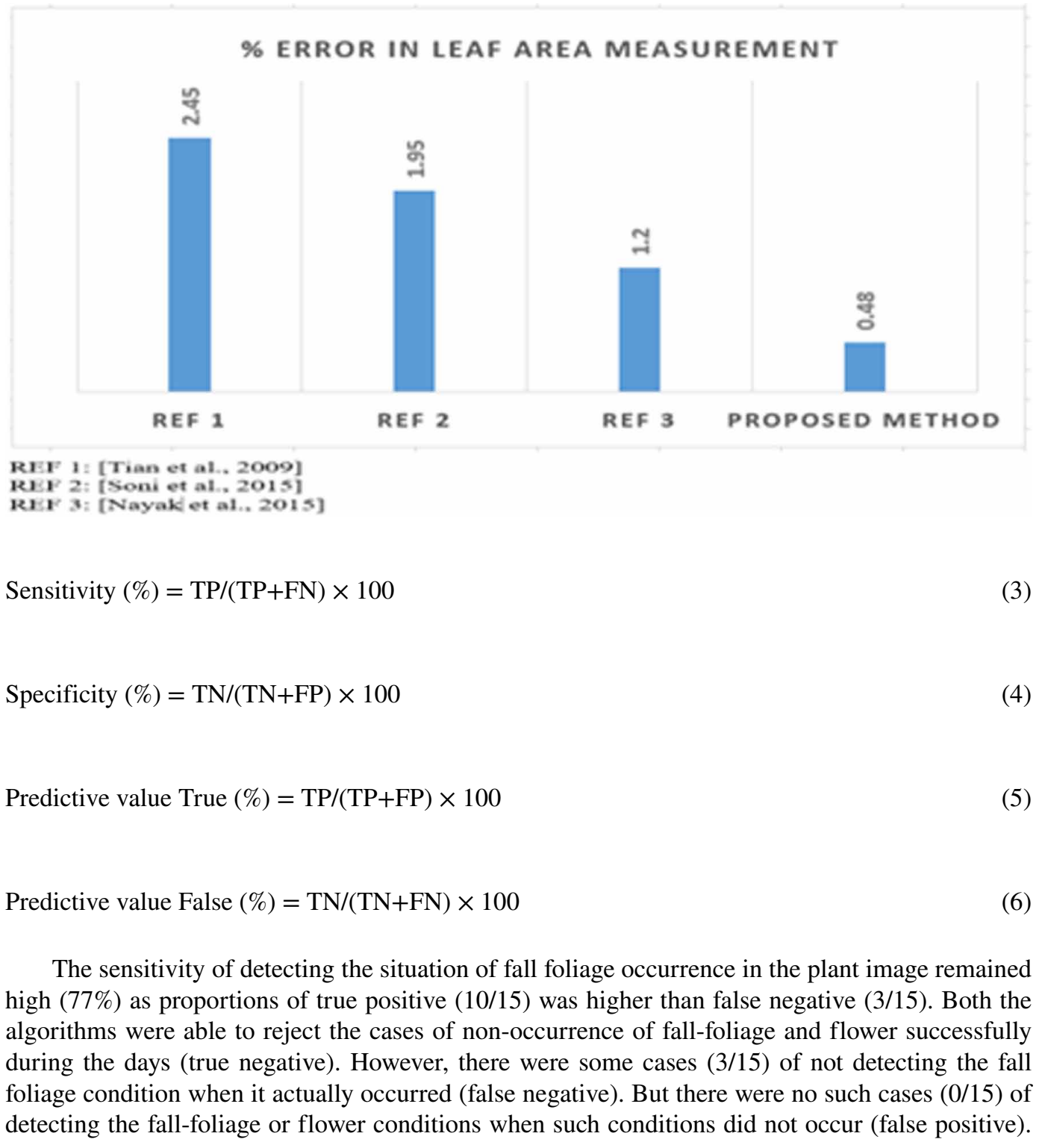


Table 1. Estimated and actual values of morphological features of a 'test' plant on different days along with statistical analysis of data

\begin{tabular}{|c|c|c|c|c|c|c|c|c|c|c|c|c|c|}
\hline \multirow[t]{2}{*}{ S.No } & \multirow[t]{2}{*}{ Date } & \multicolumn{3}{|c|}{ Plant Height $(\mathrm{cm})$} & \multicolumn{3}{|c|}{ Leaf Area $\left(\mathrm{mm}^{2}\right)$} & \multicolumn{3}{|c|}{$\begin{array}{l}\text { Fall Foliage } \\
\text { Detection }\end{array}$} & \multicolumn{3}{|c|}{ Flower Detection } \\
\hline & & 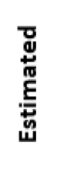 & 焉 & $\begin{array}{l}\text { ᄒे } \\
\text { யँ } \\
\text { ๙ீ }\end{array}$ & 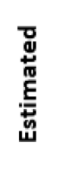 & 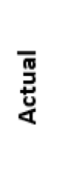 & $\begin{array}{l}\text { tั } \\
\text { ஸे } \\
\text { வீ }\end{array}$ & 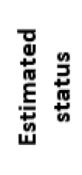 & 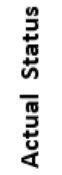 & 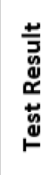 & 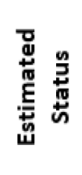 & 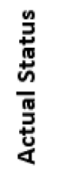 & 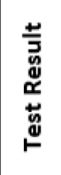 \\
\hline 1 & $24 / 05 / 16$ & 24.6 & 24.3 & 1.2 & 980 & 975 & 0.5 & NO & NO & TN & NO & NO & TN \\
\hline 2 & $25 / 05 / 16$ & 24.9 & 24.7 & 0.8 & 986 & 982 & 0.4 & NO & NO & TN & NO & NO & TN \\
\hline 3 & $26 / 05 / 16$ & 25.2 & 25.3 & -0.4 & 1000 & 991 & 0.9 & YES & YES & TP & NO & NO & TN \\
\hline 4 & $27 / 05 / 16$ & 25.7 & 25.8 & -0.4 & 1006 & 998 & 0.8 & YES & YES & TP & NO & $\mathrm{NO}$ & TN \\
\hline 5 & $28 / 05 / 16$ & 26.2 & 26.4 & -0.8 & 1012 & 1006 & 0.6 & YES & YES & TP & NO & NO & TN \\
\hline 6 & $29 / 05 / 16$ & 27.8 & 27.9 & -0.4 & 1017 & 1012 & 0.5 & NO & YES & FN & NO & $\mathrm{NO}$ & $\mathrm{TN}$ \\
\hline 7 & $30 / 05 / 16$ & 28.5 & 28.6 & -0.3 & 1028 & 1023 & 0.5 & YES & YES & TP & NO & NO & TN \\
\hline 8 & $31 / 05 / 16$ & 28.9 & 29.1 & -0.7 & 1032 & 1028 & 0.4 & NO & YES & FN & NO & $\mathrm{NO}$ & TN \\
\hline 9 & $01 / 06 / 16$ & 29.7 & 29.6 & 0.3 & 1040 & 1037 & 0.3 & YES & YES & TP & NO & $\mathrm{NO}$ & TN \\
\hline 10 & $02 / 06 / 16$ & 30.1 & 30.2 & -0.3 & 1045 & 1041 & 0.4 & YES & YES & TP & NO & NO & TN \\
\hline 11 & $03 / 06 / 16$ & 30.4 & 30.8 & -1.3 & 1052 & 1050 & 0.2 & NO & YES & FN & No & NO & TN \\
\hline 12 & $04 / 06 / 16$ & 31.8 & 31.6 & 0.6 & 1061 & 1059 & 0.2 & YES & YES & TP & NO & NO & TN \\
\hline 13 & $05 / 06 / 16$ & 32.5 & 32.3 & 0.6 & 1072 & 1065 & 0.7 & YES & YES & TP & NO & NO & $\mathrm{TN}$ \\
\hline 14 & $06 / 06 / 16$ & 33.0 & 32.9 & 0.3 & 1084 & 1080 & 0.4 & YES & YES & TP & NO & $\mathrm{NO}$ & TN \\
\hline 15 & $07 / 06 / 16$ & 33.6 & 33.5 & 0.3 & 1095 & 1089 & 0.6 & YES & YES & TP & NO & NO & TN \\
\hline
\end{tabular}

Where, TP: True Positive, TN: True Negative, FP: False Positive, and FN: False Negative.

Hence, the specificity of successfully rejecting the non-occurrence of the fall-foliage and flowers remained $100 \%$. Since during the monitoring days there was no onset of flowers, true positive or false negative cases remained zero. Both the fall-foliage and flower detection algorithms have high degree of insensitivity to false alarms

\section{CONCLUSION}

In this work, a unique machine vision tool with easy-to-use plant user interface for online/offline estimating of certain plant growth related morphological parameters has been successfully implemented. Wirelessly captured plant images using high resolution cameras are logged, processed and analyzed using image processing algorithms to estimate plant height, average leaf area, detection of flowers and fall-foliage condition within the plant. The results obtained are statistically analyzed and validated using standard methods. The working of the system in real-time conditions is found satisfactory. The successfully captured plant images are processed with minimum time lag. Software takes 1-2s to process and analyze captured plant images to provide the best estimate of the desired morphological parameters on the GUI. The information on the panel is updated at the fast rate whenever new images are taken and loaded. In automatic mode, the image acquisition time can be set as per the requirements. Generally, plant morphological parameters are measured on day/week basis to access plant development. 
Figure 10. Day variations in estimated and actual values of morphological growth parameters of the plant (a) Plant height (b) Leaf area (c) Related percentage error curves

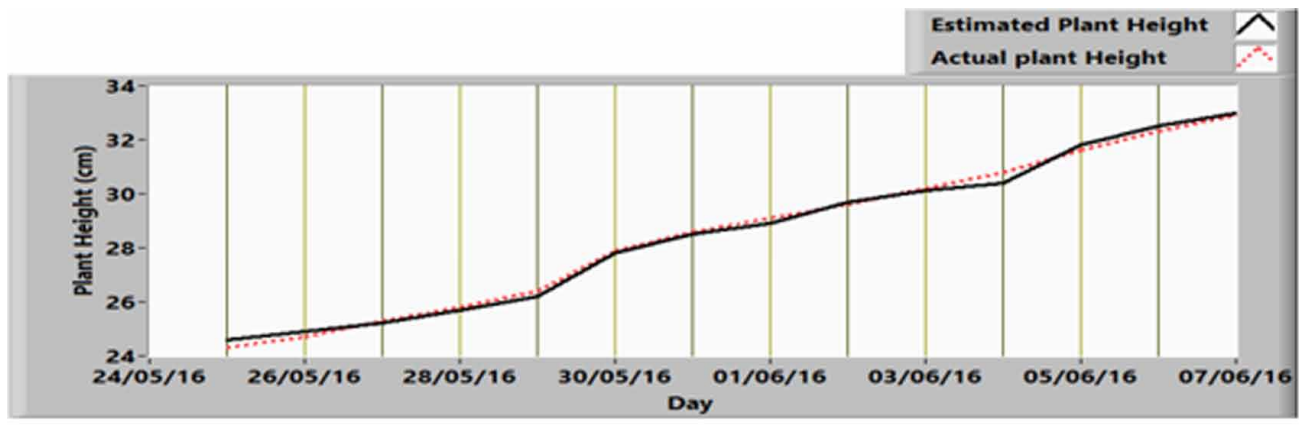

(a)

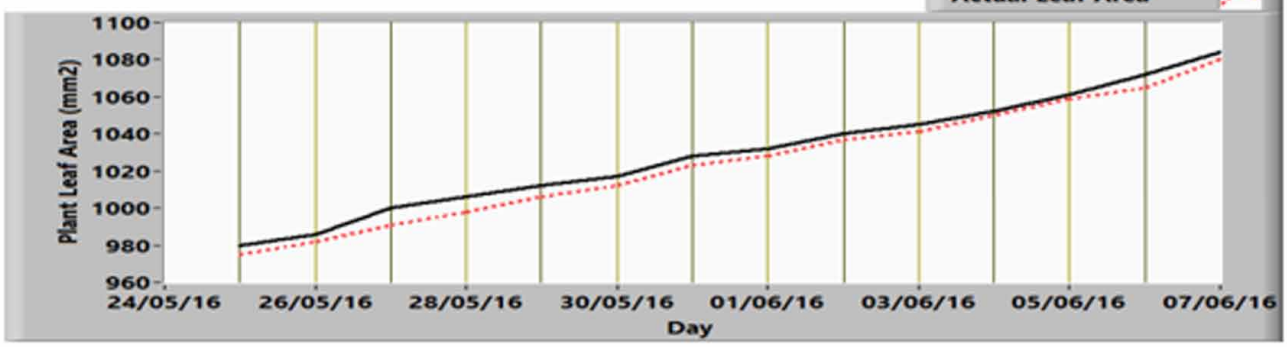

(b)

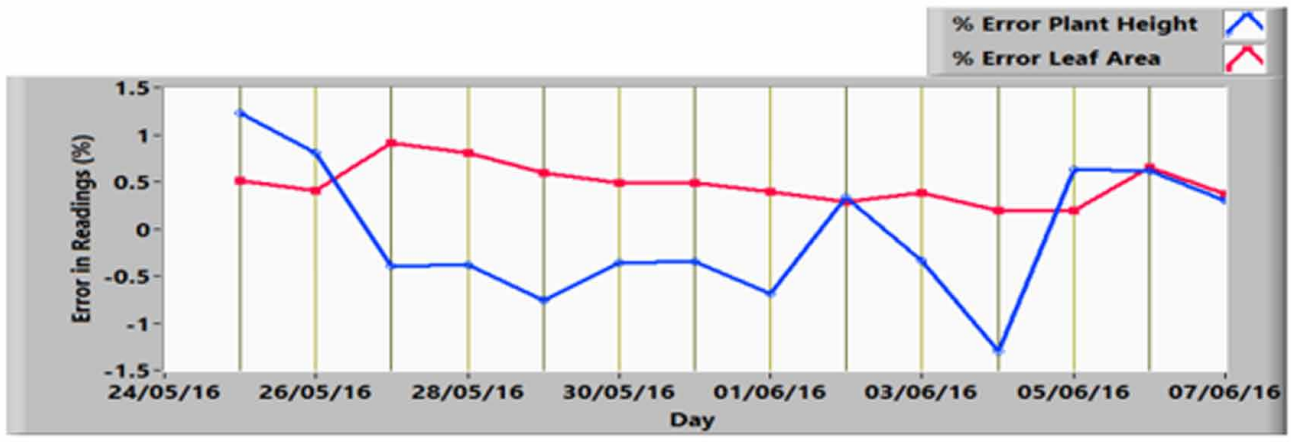

(c)

Figure 11. Test results of identifying plant morphological features (a) Fall foliage detection (b) Flower detection
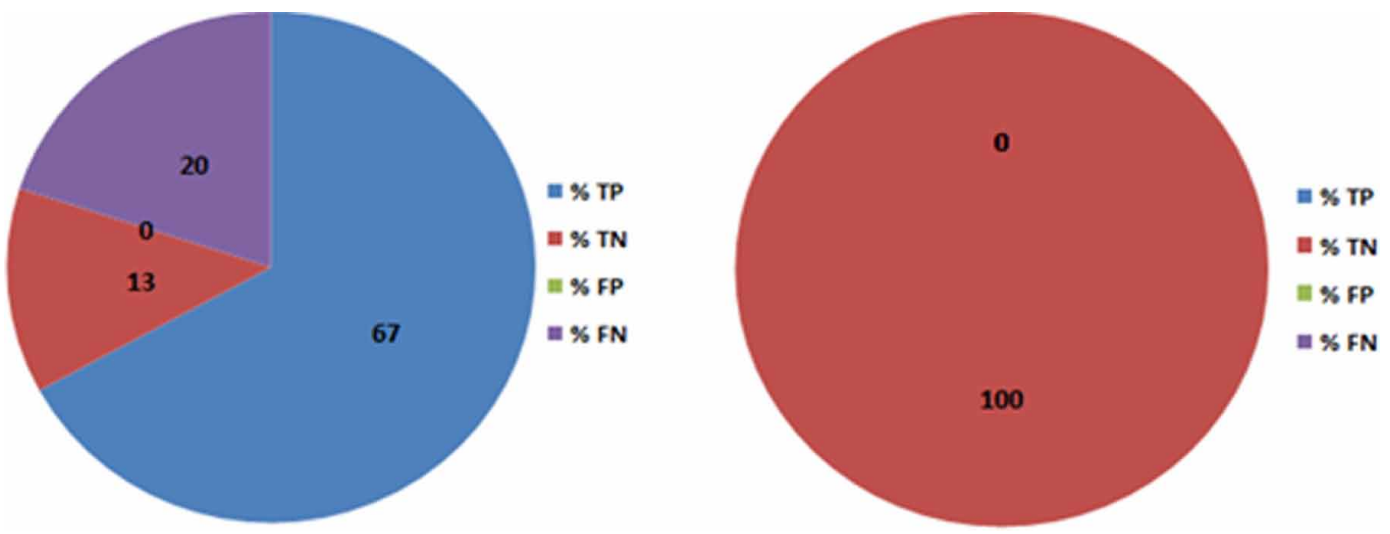


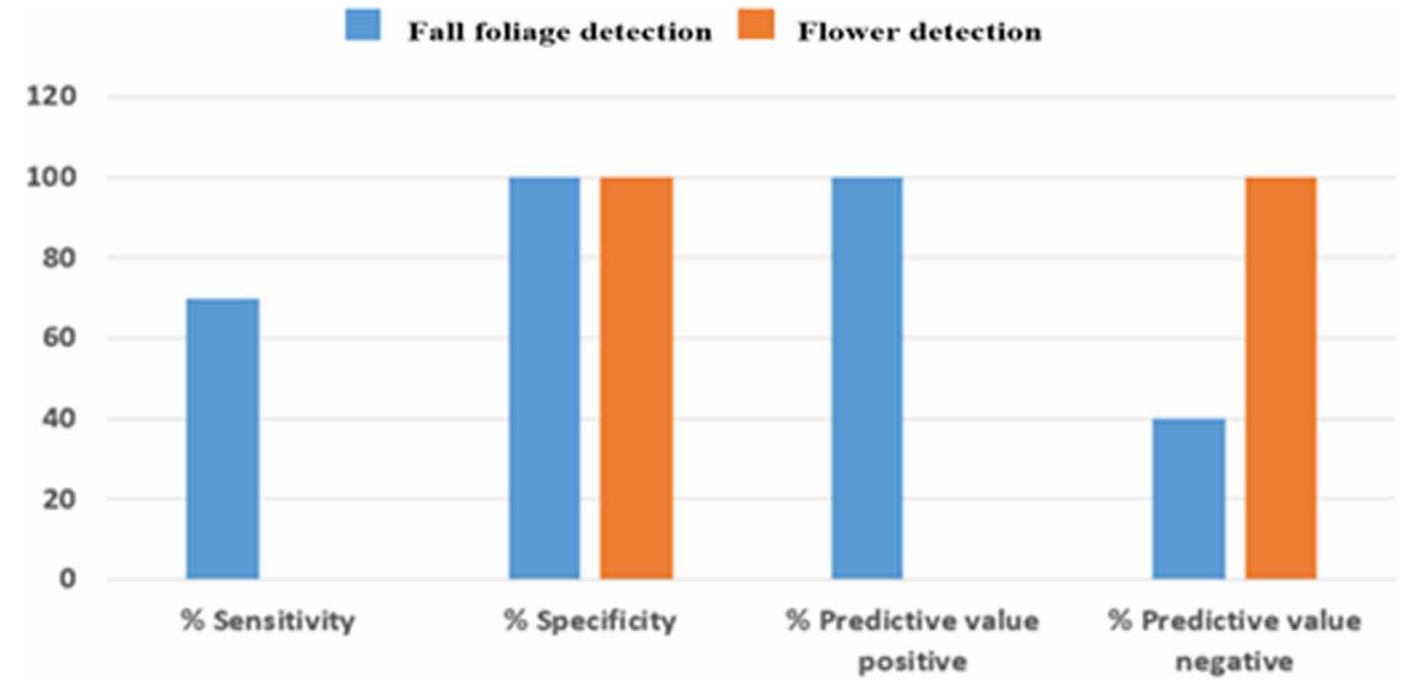

The overall measurement accuracy of all parameters remains high. The tool supports measurement of leaf area of plant with improved level of accuracy with mean percentage error of $0.48 \%$ and plant height measurement with mean percentage error of $-0.02 \%$. Both fall foliage and flower onset conditions were detected with high level of sensitivity (above 77\%) and specificity (100\%). The image analysis algorithms were able to reject the cases of non-occurrence of fall-foliage and flower successfully during the days with $100 \%$ of true negative. However, there were some cases of not detecting the fall foliage condition when it actually occurred thus providing false negative percentage of $20 \%$.

In future, the overall functionality of the system can further be enhanced by including methods of measurement of other parameters such as detection and count, occurrence of pests etc. for growth and health analysis and plant identification. The system reliability can be improved by analysing and fusing plant images from various angles using multiple cameras or rotating the camera. Further, the system being on the network can be easily integrated as Internet of Things system to analyse and classify plant growth and diseases. 


\section{REFERENCES}

Basnet, B. \& Bang, J. (2018). The state-of-the-art of knowledge-intensive agriculture: A review on applied sensing systems and data analytics. Hindawi Journal of Sensors,1-13.

Boykov, Y. Y., \& Jolly, M. P. (2001). Interactive graph cuts for optimal boundary and region segmentation of objects in N-D images. In Proceedings of the 8th International Conference on Computer Vision (Vol. 1, pp. 105-112). doi:10.1109/ICCV.2001.937505

Chen, C. H. (2013). Computer vision in Medical Imaging. River Edge, NJ: World Scientific Publishing Corporation.

Crane, R. (1997). A simplified approach to image processing. Prentice Hall PTR.

Crowe, T. G., \& Delwiche, M. J. (1996). Real-time defect detection in fruit-Part I and II: Design concepts and development of prototype hardware. Transactions of the ASAE. American Society of Agricultural Engineers, 39(6), 2299-2317. doi:10.13031/2013.27740

Teledyne Dalsa. (2014). Basics of understanding machine vision.

Das, M., Manmatha, R., \& Riseman, E. M. (1999). Indexing flower patent images using domain knowledge. IEEE Intelligent Systems, 14(5), 24-33. doi:10.1109/5254.796084

Davies, E. R. (2012). Machine vision: Theory, algorithms, practicalities. Academic Press.

Demin, Z., Wei, C., \& Lijing, L. (2012). An image processing based system for leaf parameter measuring. In Proceedings of the Symposium on Photonics and Optoelectronics, Shanghai, China. doi:10.1109/ SOPO.2012.6270478

Fukuda, K., Takiguchi, T., \& Ariki, Y. (2008). Multiple classifier based on fuzzy c-means for a flower image retrieval. In Proceedings of the International Workshop on Nonlinear Circuits and Signal Processing (pp. 76-79).

Gavhale, K. R., Gawande, U., \& Kamal, O. (2014). Unhealthy region of citrus leaf detection using image processing techniques, In Proceedings of the IEEE International Conference for Convergence of Technology, Pune, India (pp. 1-6). doi:10.1109/I2CT.2014.7092035

Goudriaan, J., \& Monteith, J. L. (1990). A mathematical function for crop growth based on light interception and leaf area expansion. Annals of Botany, 66(6), 695-701. doi:10.1093/oxfordjournals.aob.a088084

Heady, H. F. (1957). The measurement and value of plant height in the study of Herbaceous vegetation. Ecology, 38(2), 313-320. doi:10.2307/1931691

Hong, A., Chen, G., Li, J., Chi, Z., \& Zhang, D. (2004). A flower image retrieval method based on ROI feature. Journal of Zhejiang University. Science, 5(7), 764-772. doi:10.1631/jzus.2004.0764 PMID:15495304

Hopkins, W. G. (1999). Introduction to plant physiology (2nd ed.). New York, NY: John Wiley \& Sons.

Hsu, T., Lee, C., \& Chen, L. (2010). An interactive flower image recognition system. Multimedia Tools and Applications, 53(1), 53-73. doi:10.1007/s11042-010-0490-6

ISPECT. (2010). Machine vision for safety and security. INSPECT's Branch Newsletter for Vision.

Jayaraman, P.P., Yavari, A., Georgakopoulos, D., Morshed, A., \& Zaslavsky, A., (2016) Internet of things platform for smart farming: experiences and lessons learnt. Sensors, 16(1884), 1-17.

Jingwen, W., \& Hong, L. (2012). Measurement and analysis of plant leaf area based on image processing. In Proceeding of the International Symposium on Information Technology in Medicine and Education, Hokodate (Vol. 2, pp. 1070-1074). doi:10.1109/ITiME.2012.6291485

Johnson, G. W., \& Jennings, R. (2011). LabVIEW graphical programming (4th ed.). McGraw Hill.

Kallen, H. (2016). Applications of machine vision: Quality control, cancer detection and surveillance. Unpublished doctoral thesis, Mathematical sciences, Lund University, Sweden.

Kar, S., Shrikhande, S.V. \& Babu, R.M.S. (2016). Machine vision applications for physical security, quality assurance and personnel dosimetry. BARC Newsletter, 29-32. 
Katungunya, S. K., Ding, X., \& Mashenene, J. J. (2016). Automatic recognition of handwritten digits using multi-layer sigmoid neural network. International Journal of Science and Research, 5(3), 951-955.

Key Technology Inc. (2017). Digital sorting solution for food processing.

Kuchay, S. A., \& Zargar, M. A. (2016). Analysis of growth in some cultivars of Solanum Melongena grown in Kashmir. Imperial Journal of Interdisciplinary Research, 2(5), 397-405.

Lewis, J. (2014). Introduction to machine vision. Cognex Corporation.

Li, L., Niu T. (2014). Gao Yi "Mathematical methods and applications in medical imaging 2014. In Computational and Mathematical Methods in Medicine Volume, 1- 2.

Li, X., Ma, H., Wang, X., \& Zhang, X. (2018). Traffic light recognition for complex scene with fusion detections. IEEE Transactions on Intelligent Systems, 19(1), 199-208. doi:10.1109/TITS.2017.2749971

LI-COR, Inc. (1988) LI-3000A Portable Area Meter: Instruction Manual.

Lin, T.-T., Chien, C.-F., Liao, W.-C., Chung, K.-C., \& Chang, J.-M. (2006). Machine vision systems for plant growth measurement and modelling. Environment Control in Biology, 44(3), 181-187. doi:10.2525/ecb.44.181

Lü, C., Ren, H., Zhang, Y., \& Shen, Y. (2010). Leaf area measurement based on image processing. In Proceedings of the International conference on Measuring Technology and Mechatronics Automation. Changsha, China: IEEE doi:10.1109/ICMTMA.2010.141

Mahendran, R., Jayashree, G. C., \& Alagusundaram, K. (2012). Application of computer vision technique on sorting and grading of fruits and vegetables. Journal of Food Processing \& Technology, 1-7.

Najjar, A., \& Zagrouba, E. (2012). Flower image segmentation based on color analysis and a supervised evaluation. In Proceedings of the $2^{\text {nd }}$ International Conference on Communication and Information (ICCIT): Wireless Communications and Signal Processing. Hammame: IEEE doi:10.1109/ICCITechnol.2012.6285834

Nakano, K. (1997). Application of neural networks to the color grading of apples. Computers and Electronics in Agriculture, 18(2-3), 105-116. doi:10.1016/S0168-1699(97)00023-9

Nathalie, K., \& Breda, J. J. (2003). Ground-based measurements of leaf area index: A review of methods, instruments and current controversies. Journal of Experimental Botany, 54(392), 2403-2417. doi:10.1093/jxb/ erg263 PMID:14565947

National Instruments. (1999). IMAQ Vision User Manual. Part Number 322320B-01.

National Instruments. (2000a). LabVIEW User Manual. Part Number 320999C-01.

National Instruments. (2000b). LabVIEW Measurements Manual. Part Number 322661A-01.

Nayak, D., Dey, A. K., \& Sharma, M. (2015). Measurement of length and width of Betel leaf by image processing Using Matlab. In Proceedings of the International Conference on Electrical, Electronics, Signals, Communication and Optimization. Visakhapatnam, India: IEEE. doi:10.1109/EESCO.2015.7253754

Nikon Corporation. (2014). Digital Camera D 3300: User's manual.

Ojha, T., Misra, S., \& Raghuwanshi, N. S. (2015). Wireless sensor networks for agriculture: The state-of-theart in practice and future challenges. Computers and Electronics in Agriculture, 118, 66-84. doi:10.1016/j. compag.2015.08.011

Pahuja, R., Verma, H. K., \& Uddin, M. (2013). A wireless sensor network for greenhouse climate control. IEEE Pervasive Computing, 12(2), 49-58. doi:10.1109/MPRV.2013.26

Pakhira, M. K. (2011). Digital image processing and pattern recognition. PHI Learning.

Pandurng, J. A., \& Lomte, S. S. (2015). Digital image processing applications in agriculture: A Survey. Journal of Advanced Research in Computer Science and Software Engineering, 5(3), 622-624.

Parikh, R., Mathai, A., Parikh, S., Sekhar, G. C., \& Thomas, R. (2008). Understanding and using sensitivity, specificity and predictive values. Indian Journal of Ophthalmology, 56(1), 45-50. doi:10.4103/0301-4738.37595 PMID:18158403 
Patel, K. K., Kar, A., Jha, S. K., \& Khan, M. A. (2012). Machine vision system: A tool for quality inspection of food and agricultural products. Journal of Food Science and Technology, 49(2), 123-141. doi:10.1007/s13197011-0321-4 PMID:23572836

Picon, A., Bereciartua, A., Echazarra, J., Ghita, O., Whelan, P. F., \& Iriondo, P. M. (2012). Real-time hyperspectral processing for automatic nonferrous material sorting. Journal of Electronic Imaging, 21(1), 1-9. doi:10.1117/1. JEI.21.1.013018

Prasad, S., Kumar, P., \& Tripathi, R. C. (2011). Plant leaf species identification using curvelet transform. In Proceedings of the 2nd International Conference on Computer \& Communication Technology. Allahabad, India: IEEE. doi:10.1109/ICCCT.2011.6075212

Relf, C. G. (2004). Image acquisition and processing with LabVIEW. Washington, DC: CRC Press LLC.

Rosenfeld, A. (1985). Introduction to machine vision. IEEE Control Systems Magazine, 5(3), 14-17. doi:10.1109/ MCS.1985.1104954

Russ, J. C., \& Neal, B. (2017). The image processing handbook (7th ed.). CRC Press.

Saxena, L., \& Armstrong, L. L. (2014). A survey of image processing techniques for agriculture. In Proceedings of Asian Federation for Information Technology in Agriculture (pp. 401-413).

Schopfer, P. (2006). Biomechanics of plant growth. American Journal of Botany, 93(10), 1415-1425. doi:10.3732/ ajb.93.10.1415 PMID:21642088

Shafian, S., Rajan, N., Schnell, R., Bagavathiannan, M., Valasek, J., Shi, Y., \& Olsenholler, J. (2018). Unmanned aerial systems-based remote sensing for monitoring sorghum growth and development. PLoS One, 1-18. PMID:29715311

Shivling, V. D., Singla, A., Ghanshyam, C., Kapur, P., \& Gupta, S. (2011). Plant leaf imaging technique for agronomy. In Proceedings of the International Conference on Image Information Processing, Shimla, India. IEEE.

Singh, D., Vishnu, C., \& Mohan, C. K. (2016). Visual big data analytics for traffic monitoring in smart city. In Proceeding of $15^{\text {th }}$ International Conference on Machine Learning and Applications, Anaheim, CA. IEEE. doi:10.1109/ICMLA.2016.0159

Singh, M., \& Misra, A. K. (2017). Detection of plant leaf using image segmentation and soft computing. Information Processing in Agriculture, 4, 41-49. doi:10.1016/j.inpa.2016.10.005

Solari, F., Chessa, M., \& Sabatini, S. P. (Eds.). (2012). Machine vision - Applications and systems. Croatia: InTech. doi: $10.5772 / 2456$

Soni, A. P., Dey, A. K., \& Sharma, M. (2015). An image processing technique for estimation of betel leaf area. In International Conference on Electrical, Electronics, Signals, Communication and Optimization, Andhra Pradesh, India. IEEE. doi:10.1109/EESCO.2015.7253691

Susan, C. (2017). Radar remote sensing of agricultural canopies: A review. IEEE Journal of Selected Topics in Applied Earth Observations and Remote Sensing, 10(5), 2249-2273. doi:10.1109/JSTARS.2016.2639043

Szelisk, R. (2010). Computer vision: Algorithms and applications. Springer.

Tian, Y. W., \& Wang, X. J. (2009). Analysis of leaf parameters measurement of cucumber based on image processing. Software Engineering, 3, 34-37.

Tiay, T., Benyaphaichit, P., \& Riyamongkol, P. (2014). Flower recognition system based on image processing. In Proceedings of the 3rd ICT International Student Project Conference, Thailand. IEEE. doi:10.1109/ICTISPC.2014.6923227

Tsarouchi, P., Matthaiakis, S. A., Michalos, G., Makris, S., \& Chryssolouris, G. (2016). A method for detection of randomly placed objects for robotic handling CIRP. Journal of Manufacturing Science and Technology, 14, 20-27. doi:10.1016/j.cirpj.2016.04.005

Uluturk, C., \& Ugur, A. (2012). Recognition of leaves based on morphological features derived from two halfregions. In Proceedings of the International Symposium on Innovations in Intelligent Systems and Applications, Trabzon: IEEE. doi:10.1109/INISTA.2012.6247030 
Vázquez-Arellano, M., Griepentrog, H. W., Reiser, D., \& Paraforos, D. S. (2016). 3-D imaging systems for agricultural applications - A review. Sensors (Basel), 658, 1-24.

Watada, A. E, Herner, R. C., \& Kader, A. A., Romani. R. J, and Staby. (1984). Terminology for the description of developmental stages of horticultural crops. Horticultural Science, 19(1), 20-21.

Xiao-dong, Z., \& Xiao-jie, W. (2009). Feature extraction of plant leaf based on visual consistency. In Proceedings of the 1st International Symposium on Computer Network and Multimedia Technology, Wuhan. IEEE.

Yin, X., Goudriaan, J., Lantinga, E. A., Vos, J., \& Spiertz, H. J. (2003). A flexible sigmoid function of determinate growth. Annals of Botany, 91(3), 361-371. doi:10.1093/aob/mcg029 PMID:12547689

Yu, N., Li, L., Schmitz, N., Tian, L. F., Greenberg, J. A., \& Diers, B. W. (2016). Development of methods to improve soybean yield estimation and predict plant maturity with an unmanned aerial vehicle based platform. Remote Sensing of Environment, 187, 91-101. doi:10.1016/j.rse.2016.10.005

Zhao, H., \& Mi, Y. (2010). Development of an Automatic Grading System for Green Hawthorn Leaf Using Image Processing. 2010. In Proceeding of the 3rd International Congress on Image and Signal Processing. Yanti, China. IEEE. doi:10.1109/CISP.2010.5647720

Himanshu Gupta was born in Shahjahanpur, India in 1992. He received his B.Tech degree in Electronics \& Communication Engineering from Uttar Pradesh Technical University, Lucknow, India in 2014 and the M. Tech degree in Control and Instrumentation Engineering from $\operatorname{Dr} B$ R Ambedkar National Institute of Technology Jalandhar, India in 2016. He is currently working as an Assistant Professor in Department of Electronics \& Communication Engineering, MITRC Alwar, India. His current research interest includes image processing, embedded system and virtual instrumentation

Roop Pahuja is an Associate Professor in the Department of Instrumentation and Control Engineering at Dr B $R$ Ambedkar National Institute of Technology Jalandhar, India. Pahuja received M. Tech degree in Measurement and Instrumentation from the Indian Institute of Technology, Roorkee, India. She has done her PhD from National Institute of Technology Jalandhar, India, in the area of application of wireless sensor network and developed a novel system for greenhouse automation. Her research interests include design and development of intelligent virtual instrumentation, smart sensors and network systems, and WSN and its applications. She has guided more than $30 \mathrm{~B}$. Tech projects and $15 \mathrm{M}$. Tech dissertations in the area of virtual instrumentation for measurement, control or vision-based applications. She has more than 35 publications in international journals and conferences of repute. 